\title{
Assessing land-ocean connectivity via Submarine Groundwater Discharge (SGD) in the Ria Formosa Lagoon (Portugal): combining radon measurements and stable isotope hydrology
}

\section{Rocha et al.}

Correspondence to: C. Rocha (rochac@tcd.ie)

The copyright of individual parts of the supplement might differ from the CC-BY 3.0 licence. 
Table S1. Dataset of stable isotopes in water used in the study, including historical data (Carreira 1991). The table includes sample name or designation (Site), Geographical location, the date of collection, salinity of the sample (when available), the number of replicates collected and analyzed $(n)$, and observations to help locate the information in either space (within/outside the lagoon, for example) or timing with regards to the tide.

\begin{tabular}{|c|c|c|c|c|c|c|c|c|c|c|c|}
\hline Site & Lat $(\mathrm{N})$ & Lon $(\mathrm{W})$ & Date & Salinity & $\begin{array}{c}\delta^{18} \mathrm{O} \\
(\% \mathrm{VSMOW}) \\
\end{array}$ & $\begin{array}{c} \pm \text { s.d. } \\
(\% \text { VSMOW) }\end{array}$ & $\mathrm{n}$ & $\begin{array}{c}\delta^{2} \mathrm{H} \\
(\% \mathrm{VSMOW})\end{array}$ & $\begin{array}{c} \pm \text { s.d. } \\
(\% \text { VSMOW) }\end{array}$ & $\mathrm{n}$ & Observations \\
\hline \multicolumn{12}{|l|}{ Seawater } \\
\hline Southern coast & - & - & 1982 & - & 0.98 & 0.28 & 28 & 4.47 & 2.67 & 10 & Carreira (1991) \\
\hline SE coast & $36^{\circ} 59^{\prime} 16^{\prime \prime}$ & $7^{\circ} 46^{\prime} 28^{\prime \prime}$ & $05 / 12 / 2009$ & 35.69 & 1.43 & 0.52 & 3 & 5.17 & 0.88 & 3 & This Study \\
\hline SW coast & $37^{\circ} 03^{\prime} 03^{\prime \prime}$ & $8^{\circ} 07^{\prime} 13^{\prime \prime}$ & $01 / 12 / 2009$ & 35.20 & 1.30 & 0.16 & 3 & 4.8 & 0.50 & 3 & This Study \\
\hline \multicolumn{12}{|l|}{ Groundwater } \\
\hline Average & - & - & 1982 & & -4.4 & 0.15 & 25 & -25.8 & 1.55 & 25 & Carreira (1991) \\
\hline Ramalhete & $37^{\circ} 00^{\prime} 22^{\prime \prime}$ & $7^{\circ} 58^{\prime} 05^{\prime \prime}$ & $01 / 2007$ & 5.5 & -4.00 & 0.01 & 2 & -23.85 & 1.0 & 2 & Inside lagoon, $\mathrm{W}$ sector \\
\hline Montenegro & $37^{\circ} 02^{\prime} 08^{\prime \prime}$ & $7^{\circ} 57^{\prime} 41^{\prime \prime}$ & $01 / 2007$ & 0 & -4.62 & 0.01 & 2 & -27.60 & 1.0 & 2 & Coastal plain, W sector \\
\hline Gambelas & $37^{\circ} 02^{\prime} 36^{\prime \prime}$ & $7^{\circ} 58^{\prime} 34^{\prime \prime}$ & $01 / 2007$ & 0 & -4.67 & 0.02 & 2 & -27.79 & 1.0 & 2 & Coastal plain, W sector \\
\hline Bela Salema & $37^{\circ} 04^{\prime} 33^{\prime \prime}$ & $7^{\circ} 55^{\prime} 57^{\prime \prime}$ & $01 / 2007$ & 0 & -4.52 & 0.00 & 2 & - & - & - & Coastal plain, W sector \\
\hline Sta Barbara Nexe & $37^{\circ} 06^{\prime} 56^{\prime \prime}$ & $7^{\circ} 56^{\prime} 00^{\prime \prime}$ & $01 / 2007$ & 0 & -4.51 & 0.00 & 2 & -24.27 & 1.0 & 2 & $\mathrm{~N}$ of coastal Plain \\
\hline Rio Seco & $37^{\circ} 10^{\prime} 34^{\prime \prime}$ & $7^{\circ} 54^{\prime} 51^{\prime \prime}$ & $06 / 12 / 2009$ & 1.85 & -3.46 & 0.28 & 3 & -22.6 & 0.5 & 3 & Coastal plain, W sector \\
\hline Chelote & $37^{\circ} 03^{\prime} 06^{\prime \prime}$ & $7^{\circ} 55^{\prime} 30^{\prime \prime}$ & $06 / 12 / 2009$ & 1.06 & -3.73 & 0.20 & 3 & -23.5 & 0.3 & 3 & Coastal plain, W sector \\
\hline Casa Costa & $37^{\circ} 06^{\prime} 19^{\prime \prime}$ & $7^{\circ} 59^{\prime} 39^{\prime \prime}$ & $06 / 12 / 2009$ & 0.45 & -4.28 & 0.16 & 3 & -25.9 & 0.3 & 3 & Coastal plain, W sector \\
\hline Ramalhete & $37^{\circ} 00^{\prime} 22^{\prime \prime}$ & $7^{\circ} 58^{\prime} 05^{\prime \prime}$ & 08/12/2009 & 4.89 & -3.78 & 0.23 & 3 & -21.9 & 0.3 & 3 & Inside lagoon, $\mathrm{W}$ sector \\
\hline Ramalhete & $37^{\circ} 00^{\prime} 22^{\prime \prime}$ & $7^{\circ} 58^{\prime} 05^{\prime \prime}$ & $08 / 12 / 2009$ & 4.78 & -4.01 & 0.17 & 3 & -22.5 & 0.4 & 3 & Inside lagoon, W sector \\
\hline Deserta & $36^{\circ} 58^{\prime} 08^{\prime \prime}$ & $7^{\circ} 52^{\prime} 27^{\prime \prime}$ & $04 / 12 / 2009$ & 0.61 & -3.83 & 0.26 & 3 & -25.8 & 0.7 & 3 & Barrier Islands \\
\hline Deserta & $36^{\circ} 58^{\prime} 08^{\prime \prime}$ & $7^{\circ} 52^{\prime} 27^{\prime \prime}$ & $04 / 12 / 2009$ & 1.59 & -3.58 & 0.32 & 3 & -24.9 & 0.8 & 3 & Barrier Islands \\
\hline Rio Seco & $37^{\circ} 01^{\prime} 34^{\prime \prime}$ & $7^{\circ} 54^{\prime} 51^{\prime \prime}$ & $08 / 12 / 2010$ & 1.05 & -3.84 & 0.17 & 3 & -17.5 & 1.4 & 3 & Coastal plain, E sector \\
\hline Pechao Serra & $37^{\circ} 04^{\prime} 12^{\prime \prime}$ & $7^{\circ} 52^{\prime} 27^{\prime \prime}$ & $08 / 12 / 2010$ & 1.0 & -4.46 & 0.16 & 3 & -21.7 & 0.6 & 3 & Coastal plain, E sector \\
\hline Pechao Gimno & $37^{\circ} 03^{\prime} 36^{\prime \prime}$ & $7^{\circ} 52^{\prime} 15^{\prime \prime}$ & $08 / 12 / 2010$ & 1.0 & -5.09 & 0.26 & 3 & -24.8 & 1.5 & 3 & Coastal plain, E sector \\
\hline Zona industrial & $37^{\circ} 02^{\prime} 05^{\prime \prime}$ & $7^{\circ} 54^{\prime} 00^{\prime \prime}$ & $08 / 12 / 2010$ & 1.02 & -4.32 & 0.15 & 3 & -21.5 & 0.7 & 3 & Coastal plain, E sector \\
\hline \multicolumn{12}{|l|}{ Porewater } \\
\hline $\mathrm{Pw}$ a & $37^{\circ} 00^{\prime} 32^{\prime \prime}$ & $7^{\circ} 59^{\prime} 42^{\prime \prime}$ & $01 / 2007$ & 36.0 & 0.8 & 0.1 & 2 & 5.5 & 2.0 & 2 & Spring tides \\
\hline $\mathrm{Pw}_{-} \mathrm{b}$ & $37^{\circ} 00^{\prime} 26^{\prime \prime}$ & $7^{\circ} 59^{\prime} 34^{\prime \prime}$ & $01 / 2007$ & 35.5 & 0.5 & 0.1 & 2 & 8.2 & 0.8 & 2 & Spring Tides \\
\hline $\mathrm{Pw}_{\mathrm{c}}$ & $37^{\circ} 00^{\prime} 21^{\prime \prime}$ & $7^{\circ} 59^{\prime} 24^{\prime \prime}$ & $01 / 2007$ & 34.5 & 0.4 & 0.1 & 2 & 3.3 & 0.05 & 2 & Spring Tides \\
\hline Pw_d & $37^{\circ} 00^{\prime} 14^{\prime \prime}$ & $7^{\circ} 59^{\prime} 15^{\prime \prime}$ & $01 / 2007$ & 34.5 & 0.3 & 0.1 & 2 & 3.5 & 1.8 & 2 & Spring Tides \\
\hline $\mathrm{Pw}^{-} \mathrm{e}$ & $37^{\circ} 00^{\prime} 03^{\prime \prime}$ & $7^{\circ} 58^{\prime} 56^{\prime \prime}$ & $01 / 2007$ & 23.0 & -1.3 & 0.1 & 2 & -7.3 & 1.6 & 2 & Spring Tides \\
\hline $\mathrm{Pw}_{-} \mathrm{f}$ & $37^{\circ} 00^{\prime} 01^{\prime \prime}$ & $7^{\circ} 58^{\prime} 52^{\prime \prime}$ & $01 / 2007$ & 21.0 & -1.4 & 0.1 & 2 & -8.4 & 1.9 & 2 & Spring Tides \\
\hline
\end{tabular}




\begin{tabular}{|c|c|c|c|c|c|c|c|c|c|c|c|}
\hline Site & Lat (N) & Lon (W) & Date & Salinity & $\begin{array}{c}\delta^{18} \mathrm{O} \\
(\% 0 \text { VSMOW) } \\
\end{array}$ & $\begin{array}{c} \pm \text { s.d. } \\
(\% \text { VSMOW) }\end{array}$ & $\mathrm{n}$ & $\begin{array}{c}\delta^{2} \mathrm{H} \\
(\% 0 \text { VSMOW) } \\
\end{array}$ & $\begin{array}{c} \pm \text { s.d. } \\
(\% \text { VSMOW) }\end{array}$ & $\mathrm{n}$ & Observations \\
\hline Pw_h & $36^{\circ} 59^{\prime} 54^{\prime \prime}$ & $7^{\circ} 58^{\prime} 42^{\prime \prime}$ & $01 / 2007$ & 36.0 & 0.8 & 0.1 & 2 & 6.0 & 2.0 & 2 & Spring Tides \\
\hline P_B5 & $37^{\circ} 00^{\prime} 03^{\prime \prime}$ & $7^{\circ} 58^{\prime} 56^{\prime \prime}$ & $16 / 12 / 2010$ & 41.11 & -0.02 & 0.13 & 3 & 4.3 & 0.7 & 3 & Neap tides \\
\hline P_D3 & $37^{\circ} 00^{\prime} 03^{\prime \prime}$ & $7^{\circ} 58^{\prime} 56^{\prime \prime}$ & $16 / 12 / 2010$ & 42.79 & 0.22 & 0.10 & 3 & 6.3 & 0.5 & 3 & Neap Tides \\
\hline P_D5 & $37^{\circ} 00^{\prime} 03^{\prime \prime}$ & $7^{\circ} 58^{\prime} 56^{\prime \prime}$ & $16 / 12 / 2010$ & 43.05 & 0.55 & 0.12 & 3 & 7.3 & 0.8 & 3 & Neap Tides \\
\hline $\mathrm{P} \_\mathrm{A} 2$ & $37^{\circ} 00^{\prime} 03^{\prime \prime}$ & $7^{\circ} 58^{\prime} 56^{\prime \prime}$ & $16 / 12 / 2010$ & 39.05 & 0.82 & 0.18 & 3 & 6.7 & 0.7 & 3 & Neap Tides \\
\hline $\mathrm{P}_{-}^{-} \mathrm{A} 2$ & $37^{\circ} 00^{\prime} 03^{\prime}$ & $7^{\circ} 58^{\prime} 56^{\prime \prime}$ & $16 / 12 / 2010$ & 38.98 & 0.04 & 0.20 & 3 & 2.5 & 1.1 & 3 & Neap Tides \\
\hline P_A2 & $37^{\circ} 00^{\prime} 03^{\prime \prime}$ & $7^{\circ} 58^{\prime} 56^{\prime \prime}$ & $04 / 01 / 2011$ & 36.01 & 0.59 & 0.26 & 3 & 6.2 & 0.9 & 3 & Spring tides \\
\hline P_A4 & $37^{\circ} 00^{\prime} 03^{\prime \prime}$ & $7^{\circ} 58^{\prime} 56^{\prime \prime}$ & 04/01/2011 & 39.73 & -0.20 & 0.24 & 3 & 2.7 & 1.4 & 3 & Spring Tides \\
\hline $\mathrm{P}_{-}^{-} \mathrm{C} 2$ & $37^{\circ} 00^{\prime} 03^{\prime}$, & $7^{\circ} 58^{\prime} 56^{\prime \prime}$ & $04 / 01 / 2011$ & 37.35 & -0.03 & 0.21 & 3 & 5.2 & 0.8 & 3 & Spring Tides \\
\hline $\mathrm{P}+\mathrm{C} 5$ & $37^{\circ} 00^{\prime} 03^{\prime \prime}$ & $7^{\circ} 58^{\prime} 56^{\prime \prime}$ & 04/01/2011 & 38.41 & -0.08 & 0.30 & 3 & 4.6 & 2.1 & 3 & Spring Tides \\
\hline $\mathrm{P}^{-} \mathrm{E} 3$ & $37^{\circ} 00^{\prime} 03^{\prime}$, & $7^{\circ} 58^{\prime} 56^{\prime \prime}$ & $04 / 01 / 2011$ & 42.99 & -0.15 & 0.25 & 3 & 5.4 & 0.9 & 3 & Spring Tides \\
\hline P_F4 & $37^{\circ} 00^{\prime} 03^{\prime \prime}$ & $7^{\circ} 58^{\prime} 56^{\prime \prime}$ & $04 / 01 / 2011$ & 42.80 & 0.50 & 0.31 & 3 & 8.2 & 1.8 & 3 & Spring Tides \\
\hline P_B2 & $37^{\circ} 00^{\prime} 03^{\prime \prime}$ & $7^{\circ} 58^{\prime} 56^{\prime \prime}$ & $16 / 12 / 2010$ & 41.41 & 0.53 & 0.12 & 3 & 7.8 & 0.9 & 3 & Neap Tides \\
\hline P_E1 & $37^{\circ} 00^{\prime} 03^{\prime \prime}$ & $7^{\circ} 58^{\prime} 56^{\prime \prime}$ & $16 / 12 / 2010$ & 43.38 & 0.37 & 0.31 & 3 & 6.4 & 1.7 & 3 & Neap Tides \\
\hline $\mathrm{P}$-A1 & $37^{\circ} 00^{\prime} 03^{\prime \prime}$ & $7^{\circ} 58^{\prime} 56^{\prime \prime}$ & $16 / 12 / 2010$ & 40.76 & 0.62 & 0.15 & 3 & 7.9 & 0.6 & 3 & Neap Tides \\
\hline $\mathrm{P}+\mathrm{C} 1$ & $37^{\circ} 00^{\prime} 03^{\prime \prime}$ & $7^{\circ} 58^{\prime} 56^{\prime \prime}$ & $16 / 12 / 2010$ & 42.90 & 0.33 & 0.21 & 3 & 5.9 & 0.8 & 3 & Neap Tides \\
\hline $\mathrm{P}_{-}^{-} \mathrm{C} 1$ & $37^{\circ} 00^{\prime} 03^{\prime \prime}$ & $7^{\circ} 58^{\prime} 56^{\prime \prime}$ & $16 / 12 / 2010$ & 42.85 & 0.13 & 0.20 & 3 & 3.4 & 1.1 & 3 & Neap Tides \\
\hline P_A1 & $37^{\circ} 00^{\prime} 03^{\prime}$ & $7^{\circ} 58^{\prime} 56^{\prime \prime}$ & $16 / 12 / 2010$ & 41.31 & 0.64 & 0.27 & 3 & 6.1 & 2.2 & 3 & Neap Tides \\
\hline P F4 & $37^{\circ} 00^{\prime} 03^{\prime}$ & $7^{\circ} 58^{\prime} 56^{\prime \prime}$ & $12 / 01 / 2011$ & 42.07 & 0.87 & 0.28 & 3 & 8.4 & 1.4 & 3 & Spring Tides \\
\hline $\mathrm{P}+\mathrm{B} 5$ & $37^{\circ} 00^{\prime} 03^{\prime \prime}$ & $7^{\circ} 58^{\prime} 56^{\prime \prime}$ & $12 / 01 / 2011$ & 38.76 & 0.96 & 0.24 & 3 & 8.5 & 2.2 & 3 & Spring Tides \\
\hline P_A1 & $37^{\circ} 00^{\prime} 03^{\prime \prime}$ & $7^{\circ} 58^{\prime} 56^{\prime \prime}$ & $12 / 01 / 2011$ & 37.34 & 0.82 & 0.24 & 3 & 8.1 & 1.1 & 3 & Spring Tides \\
\hline P_D3 & $37^{\circ} 00^{\prime} 03^{\prime \prime}$ & $7^{\circ} 58^{\prime} 56^{\prime \prime}$ & $12 / 01 / 2011$ & 40.24 & 0.49 & 0.20 & 3 & 6.1 & 1.0 & 3 & Spring Tides \\
\hline P_D5 & $37^{\circ} 00^{\prime} 03^{\prime \prime}$ & $7^{\circ} 58^{\prime} 56^{\prime \prime}$ & $12 / 01 / 2011$ & 38.64 & 0.94 & 0.41 & 3 & 7.8 & 2.4 & 3 & Spring Tides \\
\hline \multicolumn{12}{|l|}{$\begin{array}{l}\text { Surface water } \\
\text { Lagoon }\end{array}$} \\
\hline Qta. do Lago & $37^{\circ} 01^{\prime} 49^{\prime \prime}$ & $8^{\circ} 01^{\prime} 36^{\prime \prime}$ & $07 / 2007$ & 22.0 & -0.2 & 0.1 & 2 & -3.4 & 0.6 & 2 & Flood tide \\
\hline $3 \mathrm{~B}$ & $37^{\circ} 00^{\prime} 08^{\prime \prime}$ & $7^{\circ} 58^{\prime} 48^{\prime \prime}$ & 07/2007 & 36.0 & 0.8 & 0.1 & 2 & 6.5 & 0.05 & 2 & Flood tide \\
\hline 2 & $36^{\circ} 59^{\prime} 49^{\prime \prime}$ & $7^{\circ} 55^{\prime} 49^{\prime \prime}$ & $07 / 2007$ & 36.0 & 0.9 & 0.05 & 2 & 6.2 & 1.2 & 2 & Flood tide \\
\hline 3 & $37^{\circ} 00^{\prime} 03^{\prime \prime}$ & $7^{\circ} 55^{\prime} 01^{\prime \prime}$ & 07/2007 & 36.0 & 0.9 & 0.1 & 2 & 7.3 & 0.6 & 2 & Flood tide \\
\hline 4 & $36^{\circ} 59^{\prime} 23^{\prime \prime}$ & $7^{\circ} 54^{\prime} 07^{\prime}$ & $07 / 2007$ & 35.5 & 0.8 & 0.05 & 2 & 6.3 & 0.2 & 2 & Flood tide \\
\hline A & $36^{\circ} 58^{\prime} 37^{\prime \prime}$ & $7^{\circ} 52^{\prime} 20^{\prime \prime}$ & $07 / 2007$ & 35.5 & 0.8 & 0.05 & 2 & 6.0 & 0.7 & 2 & Flood tide \\
\hline \multicolumn{12}{|l|}{$W$ Sector, $L T$} \\
\hline 1 & $37^{\circ} 00^{\prime} 28^{\prime \prime}$ & $7^{\circ} 56^{\prime} 24^{\prime \prime}$ & $02 / 12 / 2009$ & 36.09 & 1.61 & 0.17 & 3 & 7.1 & 0.7 & 3 & Low tide slack \\
\hline 2 & $36^{\circ} 59^{\prime} 49^{\prime \prime}$ & $7^{\circ} 55^{\prime} 49^{\prime \prime}$ & $02 / 12 / 2009$ & 34.75 & 2.04 & 0.37 & 3 & 7.9 & 0.6 & 3 & Low tide slack \\
\hline
\end{tabular}




\begin{tabular}{|c|c|c|c|c|c|c|c|c|c|c|c|}
\hline Site & Lat $(\mathrm{N})$ & Lon (W) & Date & Salinity & $\begin{array}{c}\delta^{18} \mathrm{O} \\
\text { (\%o VSMOW) } \\
\end{array}$ & $\begin{array}{c} \pm \text { s.d. } \\
\text { (\%o VSMOW) }\end{array}$ & $\mathrm{n}$ & $\begin{array}{c}\delta^{2} \mathrm{H} \\
(\% \mathrm{VSMOW})\end{array}$ & $\begin{array}{c} \pm \text { s.d. } \\
(\% \text { VSMOW) }\end{array}$ & $\mathrm{n}$ & Observations \\
\hline 3 & $37^{\circ} 00^{\prime} 03^{\prime \prime}$ & $7^{\circ} 55^{\prime} 01^{\prime \prime}$ & $02 / 12 / 2009$ & 33.57 & 2.05 & 0.43 & 3 & 7.6 & 0.5 & 3 & Low tide slack \\
\hline 4 & $36^{\circ} 59^{\prime} 23^{\prime}$, & $7^{\circ} 54^{\prime} 07^{\prime}$ & $02 / 12 / 2009$ & 33.82 & 1.78 & 0.26 & 3 & 7.1 & 0.3 & 3 & Low tide slack \\
\hline 5 & $36^{\circ} 58^{\prime} 42^{\prime \prime}$ & $7^{\circ} 52^{\prime} 57^{\prime \prime}$ & $02 / 12 / 2009$ & 33.84 & 0.64 & 0.08 & 3 & 6.6 & 0.6 & 3 & Low tide slack \\
\hline 1B & $37^{\circ} 00^{\prime} 20^{\prime \prime}$ & $7^{\circ} 56^{\prime} 44^{\prime \prime}$ & $02 / 12 / 2009$ & 34.63 & 0.53 & 0.32 & 3 & 7.3 & 1.0 & 3 & Low tide slack \\
\hline $2 \mathrm{~B}$ & $37^{\circ} 00^{\prime} 17^{\prime}$ & $7^{\circ} 57^{\prime} 49^{\prime \prime}$ & $02 / 12 / 2009$ & 35.76 & -0.82 & 0.34 & 3 & 5.3 & 0.6 & 3 & Low tide slack \\
\hline $3 \mathrm{~B}$ & $37^{\circ} 00^{\prime} 08^{\prime}$ & $7^{\circ} 58^{\prime} 48^{\prime \prime}$ & $02 / 12 / 2009$ & 35.33 & -0.75 & 0.19 & 3 & 6.2 & 0.6 & 3 & Low tide slack \\
\hline $4 \mathrm{~B}$ & $36^{\circ} 59^{\prime} 37^{\prime}$ & $7^{\circ} 58^{\prime} 03^{\prime \prime}$ & $02 / 12 / 2009$ & 33.84 & -0.69 & 0.12 & 3 & 6.4 & 0.4 & 3 & Low tide slack \\
\hline $5 \mathrm{~B}$ & $36^{\circ} 59^{\prime} 06^{\prime \prime}$ & $7^{\circ} 57^{\prime} 31^{\prime \prime}$ & $02 / 12 / 2009$ & 34.11 & -0.37 & 0.18 & 3 & 6.9 & 0.5 & 3 & Low tide slack \\
\hline \multicolumn{12}{|c|}{ W Sector, HT } \\
\hline 5 & $36^{\circ} 58^{\prime} 42^{\prime \prime}$ & $7^{\circ} 52^{\prime} 57^{\prime \prime}$ & $02 / 12 / 2009$ & 35.46 & -0.03 & 0.31 & 3 & 6.4 & 0.4 & 3 & High tide slack \\
\hline 4 & $36^{\circ} 59^{\prime} 23^{\prime}$ & $7^{\circ} 54^{\prime} 07^{\prime \prime}$ & $02 / 12 / 2009$ & 34.96 & -0.16 & 0.24 & 3 & 6.6 & 0.3 & 3 & High tide slack \\
\hline 3 & $37^{\circ} 00^{\prime} 03^{\prime}$ & $7^{\circ} 55^{\prime} 01^{\prime \prime}$ & $02 / 12 / 2009$ & 35.28 & -0.11 & 0.40 & 3 & 6.9 & 0.4 & 3 & High tide slack \\
\hline 2 & $36^{\circ} 59^{\prime} 49^{\prime}$, & $7^{\circ} 55^{\prime} 49^{\prime \prime}$ & $02 / 12 / 2009$ & 33.79 & 0.26 & 0.32 & 3 & 6.6 & 0.4 & 3 & High tide slack \\
\hline 1 & $37^{\circ} 00^{\prime} 28^{\prime \prime}$ & $7^{\circ} 56^{\prime} 24^{\prime \prime}$ & $02 / 12 / 2009$ & 34.73 & 0.43 & 0.21 & 3 & 6.9 & 0.2 & 3 & High tide slack \\
\hline $5 \mathrm{~B}$ & $36^{\circ} 59^{\prime} 06^{\prime \prime}$ & $7^{\circ} 57^{\prime} 31^{\prime \prime}$ & $02 / 12 / 2009$ & 35.86 & 0.41 & 0.33 & 3 & 7.2 & 0.2 & 3 & High tide slack \\
\hline $4 \mathrm{~B}$ & $36^{\circ} 59^{\prime} 37^{\prime \prime}$ & $7^{\circ} 58^{\prime} 03^{\prime \prime}$ & $02 / 12 / 2009$ & 35.36 & 0.58 & 0.17 & 3 & 7.3 & 0.5 & 3 & High tide slack \\
\hline $3 \mathrm{~B}$ & $37^{\circ} 00^{\prime} 08^{\prime \prime}$ & $7^{\circ} 58^{\prime} 48^{\prime \prime}$ & $02 / 12 / 2009$ & 35.56 & 0.66 & 0.22 & 3 & 5.1 & 0.7 & 3 & High tide slack \\
\hline $2 \mathrm{~B}$ & $37^{\circ} 00^{\prime} 17^{\prime}$ & $7^{\circ} 57^{\prime} 49^{\prime \prime}$ & $02 / 12 / 2009$ & 35.46 & 0.86 & 0.13 & 3 & 6.0 & 0.7 & 3 & High tide slack \\
\hline 1B & $37^{\circ} 00^{\prime} 20^{\prime \prime}$ & $7^{\circ} 56^{\prime} 44^{\prime \prime}$ & $02 / 12 / 2009$ & 34.98 & 0.86 & 0.12 & 3 & 6.6 & 0.6 & 3 & High tide slack \\
\hline \multicolumn{12}{|l|}{ E Sector, $L T$} \\
\hline A & $36^{\circ} 58^{\prime} 37^{\prime}$ & $7^{\circ} 52^{\prime} 20^{\prime \prime}$ & 05/12/2009 & 35.31 & 0.98 & 0.10 & 3 & 6.9 & 0.5 & 3 & Low tide slack \\
\hline B & $36^{\circ} 59^{\prime} 02^{\prime \prime}$ & $7^{\circ} 51^{\prime} 42^{\prime \prime}$ & 05/12/2009 & 36.78 & 0.78 & 0.21 & 3 & 6.4 & 1.1 & 3 & Low tide slack \\
\hline $\mathrm{C}$ & $37^{\circ} 00^{\prime} 16^{\prime \prime}$ & $7^{\circ} 49^{\prime} 31^{\prime \prime}$ & $05 / 12 / 2009$ & 35.96 & 0.78 & 0.10 & 3 & 6.5 & 0.6 & 3 & Low tide slack \\
\hline $\mathrm{D}$ & $37^{\circ} 00^{\prime} 50^{\prime}$ & $7^{\circ} 48^{\prime} 22^{\prime \prime}$ & 05/12/2009 & 34.29 & 0.87 & 0.17 & 3 & 6.5 & 0.7 & 3 & Low tide slack \\
\hline $\mathrm{E}$ & $37^{\circ} 02^{\prime} 00^{\prime \prime}$ & $7^{\circ} 47^{\prime} 38^{\prime \prime}$ & $05 / 12 / 2009$ & 36.11 & 0.81 & 0.18 & 3 & 6.3 & 0.9 & 3 & Low tide slack \\
\hline $\mathrm{F}$ & $36^{\circ} 59^{\prime} 55^{\prime \prime}$ & $7^{\circ} 51^{\prime} 22^{\prime \prime}$ & 05/12/2009 & 33.39 & 1.34 & 0.18 & 3 & 8.4 & 0.7 & 3 & Low tide slack \\
\hline G & $37^{\circ} 00^{\prime} 24^{\prime \prime}$ & $7^{\circ} 52^{\prime} 10^{\prime \prime}$ & $05 / 12 / 2009$ & 35.84 & 1.19 & 0.09 & 3 & 8.0 & 0.5 & 3 & Low tide slack \\
\hline $\mathrm{H}$ & $37^{\circ} 01 ' 19^{\prime \prime}$ & $7^{\circ} 50^{\prime} 11^{\prime \prime}$ & 05/12/2009 & 35.95 & 1.35 & 0.16 & 3 & 8.5 & 0.5 & 3 & Low tide slack \\
\hline $\mathrm{J}$ & $37^{\circ} 00^{\prime} 18^{\prime \prime}$ & $7^{\circ} 46^{\prime} 52^{\prime \prime}$ & $05 / 12 / 2009$ & 35.69 & 0.91 & 0.14 & 3 & 6.5 & 1.0 & 3 & Low tide slack \\
\hline \multicolumn{12}{|l|}{ E Sector, $H T$} \\
\hline $\mathrm{H}$ & $37^{\circ} 01^{\prime} 19^{\prime \prime}$ & $7^{\circ} 50^{\prime} 11^{\prime \prime}$ & 05/12/2009 & 36.46 & 0.88 & 0.18 & 3 & 4.6 & 1.2 & 3 & High tide slack \\
\hline A & $36^{\circ} 58^{\prime} 37^{\prime}$ & $7^{\circ} 52^{\prime} 20^{\prime \prime}$ & 05/12/2009 & 37.18 & 0.84 & 0.21 & 3 & 6.5 & 1.0 & 3 & High tide slack \\
\hline B & $36^{\circ} 59^{\prime} 02^{\prime}$ & $7^{\circ} 51^{\prime} 42^{\prime \prime}$ & 05/12/2009 & 35.96 & 0.91 & 0.09 & 3 & 7.0 & 0.8 & 3 & High tide slack \\
\hline $\mathrm{C}$ & $37^{\circ} 00^{\prime} 16^{\prime \prime}$ & $7^{\circ} 49^{\prime} 31^{\prime \prime}$ & $05 / 12 / 2009$ & 35.91 & 0.87 & 0.20 & 3 & 6.8 & 0.9 & 3 & High tide slack \\
\hline $\mathrm{D}$ & $37^{\circ} 00^{\prime} 50^{\prime}$ & $7^{\circ} 48^{\prime} 22^{\prime \prime}$ & 05/12/2009 & 35.61 & 0.86 & 0.21 & 3 & 7.6 & 1.4 & 3 & High tide slack \\
\hline E & $37^{\circ} 02^{\prime} 00^{\prime \prime}$ & $7^{\circ} 47^{\prime} 38^{\prime \prime}$ & 05/12/2009 & 37.84 & 0.91 & 0.08 & 3 & 7.7 & 0.8 & 3 & High tide slack \\
\hline $\mathrm{F}$ & $36^{\circ} 59^{\prime} 55^{\prime}$ & $7^{\circ} 51^{\prime} 22^{\prime \prime}$ & 05/12/2009 & 37.18 & 0.84 & 0.29 & 3 & 7.3 & 1.4 & 3 & High tide slack \\
\hline
\end{tabular}




\begin{tabular}{|c|c|c|c|c|c|c|c|c|c|c|c|}
\hline Site & Lat $(\mathrm{N})$ & Lon $(\mathrm{W})$ & Date & Salinity & $\begin{array}{c}\delta^{18} \mathrm{O} \\
(\% \mathrm{VSMOW})\end{array}$ & $\begin{array}{c} \pm \text { s.d. } \\
(\% \text { VSMOW })\end{array}$ & $\mathrm{n}$ & $\begin{array}{c}\delta^{2} \mathrm{H} \\
(\% \mathrm{VSMOW})\end{array}$ & $\begin{array}{c} \pm \text { s.d. } \\
(\% \text { VSMOW) }\end{array}$ & $\mathrm{n}$ & Observations \\
\hline G & $37^{\circ} 00^{\prime} 24^{\prime \prime}$ & $7^{\circ} 52^{\prime} 10^{\prime \prime}$ & $05 / 12 / 2009$ & 35.84 & 0.92 & 0.19 & 3 & 7.8 & 0.9 & 3 & High tide slack \\
\hline \multicolumn{12}{|l|}{ Beach drainage } \\
\hline BD_1 & $37^{\circ} 00^{\prime} 14^{\prime \prime}$ & $7^{\circ} 59^{\prime} 15^{\prime \prime}$ & 07/2007 & 35.0 & 0.8 & 0.1 & 2 & 5.8 & 0.2 & 2 & Spring Tides \\
\hline BD_2 & $37^{\circ} 00^{\prime} 03^{\prime \prime}$ & $7^{\circ} 58^{\prime} 56^{\prime \prime}$ & 07/2007 & 31.0 & 0.2 & 0.1 & 2 & 1.9 & 0.2 & 2 & Spring Tides \\
\hline $\mathrm{BD}_{-} 3$ & $37^{\circ} 00^{\prime} 03^{\prime}$, & $7^{\circ} 58^{\prime} 56^{\prime \prime}$ & 07/2007 & 35.0 & 0.8 & 0.1 & 2 & 5.6 & 0.5 & 2 & Spring Tides \\
\hline BD_G & $37^{\circ} 00^{\prime} 01^{\prime \prime}$ & $7^{\circ} 58^{\prime} 52^{\prime \prime}$ & 07/2007 & 35.0 & 0.8 & 0.1 & 2 & 5.1 & 1.2 & 2 & Spring Tides \\
\hline \multicolumn{12}{|l|}{ WWTP Faro } \\
\hline Settling Basin 1 & $37^{\circ} 01^{\prime} 02^{\prime \prime}$ & $7^{\circ} 57^{\prime} 24^{\prime \prime}$ & $07 / 2007$ & 2.00 & -3.6 & 0.05 & 2 & -20.2 & 1.0 & 2 & \\
\hline Settling Basin 2 & $37^{\circ} 00^{\prime} 58^{\prime \prime}$ & $7^{\circ} 57^{\prime} 23^{\prime \prime}$ & 07/2007 & 0.50 & -3.3 & 0.05 & 2 & -19.6 & 1.0 & 2 & \\
\hline Outlet Ramalhete & $37^{\circ} 01^{\prime} 00^{\prime \prime}$ & $7^{\circ} 57^{\prime} 27^{\prime \prime}$ & 07/2007 & 16.0 & -2.3 & 0.1 & 2 & -11.4 & 1.0 & 2 & \\
\hline \multicolumn{12}{|l|}{ Surf. reservoirs } \\
\hline Qta do Lago res. & $37^{\circ} 01^{\prime} 58^{\prime \prime}$ & $8^{\circ} 01^{\prime} 34^{\prime \prime}$ & 07/2007 & 20.0 & -0.3 & 0.05 & 2 & - & - & - & Spring Tides \\
\hline Ludo & $37^{\circ} 02^{\prime} 07^{\prime \prime}$ & $7^{\circ} 59^{\prime} 31^{\prime \prime}$ & 07/2007 & 1.0 & -3.7 & 0.05 & 2 & -21.4 & 1.0 & 2 & Ribeira de S. Lourenço \\
\hline \multicolumn{12}{|l|}{ Gilao River } \\
\hline Ponte Romana & $37^{\circ} 09^{\prime} 08^{\prime \prime}$ & $7^{\circ} 39^{\prime} 42^{\prime \prime}$ & $08 / 12 / 2010$ & 0.30 & -3.15 & 0.23 & 3 & -16.7 & 1.4 & 3 & \\
\hline Banco de Areia & $37^{\circ} 08^{\prime} 31^{\prime}$, & $7^{\circ} 39^{\prime} 25^{\prime \prime}$ & 08/12/2010 & 2.62 & -3.80 & 0.08 & 3 & -16.9 & 0.8 & 3 & \\
\hline Tavira & $37^{\circ} 06^{\prime} 36^{\prime \prime}$ & $7^{\circ} 38^{\prime} 59^{\prime \prime}$ & $08 / 12 / 2010$ & 29.58 & -0.15 & 0.12 & 3 & -1.2 & 0.3 & 3 & \\
\hline
\end{tabular}

